\title{
Relation of Plasmid DNA to Indoleacetic Acid Production in Different Strains of Pseudomonas syringae pv. savastanoi
}

\author{
By LUCA COMAI, $\dagger$ GIUSEPPE SURICO $\ddagger$ AND TSUNE KOSUGE* \\ Department of Plant Pathology, University of California, Davis, California 95616, U.S.A.
}

(Received 25 November 1981; revised 25 February 1982)

\begin{abstract}
Pseudomonas syringae pv. savastanoi is a bacterial pathogen of olive, oleander and privet, on which it induces tumorous overgrowths called knots or galls. Production of indoleacetic acid (IAA), a necessary factor for gall induction, is coded in oleander strain EW2009 by a $52 \mathrm{~kb}$ plasmid called pIAA1. To determine if this trait were commonly plasmid-borne in other strains of this pathogen, Iaa $^{-}$mutants were isolated from twelve different strains by selection for $\alpha$-methyltryptophan resistance. Each mutant was compared in its plasmid pattern with the parent wild-type. In oleander strains PB205 and PB213 loss of IAA production was associated with loss of, or large deletions in, a $73 \mathrm{~kb}$ plasmid called pIAA2. In the remaining strains from olive and privet loss of IAA production was not accompanied by the loss of any plasmid. To prove that pIAA2 coded for the genes for IAA production, a radioactive probe containing the first gene of the IAA pathway (iaaM) was hybridized to the plasmid DNA of the twelve strains. Only pIAA2 showed homology. We concluded that of all strains studied only PB205 and PB213 harboured a plasmid coding for IAA production, while in the remaining strains the IAA genes were located on the chromosome.
\end{abstract}

\section{INTRODUCTION}

In plant-associated bacteria, plasmids carrying genes necessary for successful symbiosis or parasitism have been described in Rhizobium (Hirsch et al., 1980), Agrobacterium (Watson et al., 1975) and in the 'Oleander Knot' agent (Comai \& Kosuge, 1980). Oleander Knot, caused by Pseudomonas syringae pv. savastanoi (Smith) Young, (henceforth referred to as pv. savastanoi) is a disease characterized by the development of galls on different organs of oleander plants. Galls are the result of hypertrophic and hyperplastic growth of the cells surrounding the infection site. A similar disease is induced on olive and privet. In contrast to crown gall, there is no evidence suggesting that plant cells infected by pv. savastanoi are transformed (E. E. Wilson, \& T. Kosuge, unpublished results). Rather, knot formation appears to depend on bacterial production of phytohormones. Pathovar savastanoi produces both indoleacetic acid (IAA) (Beltra, 1959) and cytokinins (Surico et al., 1975) in culture. A determining role for IAA in gall induction by oleander strains has been demonstrated (Smidt \& Kosuge, 1978; L. Comai \& T. Kosuge, unpublished results).

IAA is synthesized in two enzymic steps from L-tryptophan: conversion to indoleacetamide catalysed by tryptophan 2-monooxygenase (EC 1.13.12.3), and hydrolysis of indoleacetamide to IAA by indoleacetamide hydrolase (Kosuge et al., 1966). The genetic loci for these two enzymes are designated iaaM and $i a a H$ respectively and, in strain EW2009 (previously referred to as 2009), they are borne on a $52 \mathrm{~kb}$ plasmid called plAA1 (Comai \&

† Present address: Calgene Inc., 1910 Fifth Street, Suite F, Davis, CA 85616, U.S.A.

$\ddagger$ Present address: Istituto del C.N.R. su le Tossine e Micotossine dei Parassiti Vegetali, Istituto di Patologia Vegetale, Università degli Studi, Facolta di Agraria, 70126 Bari, Italy. 
Kosuge, 1980). These loci have been mapped on pIAAl by molecular cloning and characterization of insertion mutations (Comai \& Kosuge, 1982, and unpublished results).

The aim of this study was to determine if plasmid borne genes for IAA production were a characteristic common among pv. savastanoi strains. The pathogen is endemic in the Mediterranean region; thus, we chose to characterize a number of wild-type strains from southern Italy, which had been isolated from diseased olive, oleander and privet. According to the host from which they were isolated, these strains can usually be grouped in three subdivisions distinguishable on the basis of host range and other physiological characters (Bottalico \& Ercolani, 1971; Wilson et al., 1972).

Strains in which the IAA genes were plasmid borne were determined as follows: (i) isolation of spontaneous $\mathrm{Iaa}^{-}$mutants (IAA-non-producers) from each strain; (ii) analysis of their plasmids to determine if loss of IAA production were associated with loss or rearrangement of extrachromosomal DNA; (iii) hybridization of a probe plasmid containing the cloned iaaM locus to the plasmid DNA of each strain by Southern blot hybridization (Southern, 1975).

\section{METHODS}

Bacterial isolates. Fourteen isolates of pv. savastanoi were used. Eleven were from the collection of the Istituto di Patologia Vegetale at the University of Bari, Italy and had been isolated between 1965 and 1976 from knots of oleander (PB204, PB205, PB213), olive (PB202, PB207, PB230, PB231) and privet (PB209, PB215, PB217, PB218). The remaining three used as references or for some preliminary experiments, were olive strains EW 1006 and TK 1050 and oleander strain EW2009 from the collection of the Department of Plant Pathology, University of California, Davis. Plasmids from olive strain PB207 were used as molecular weight standards in Fig. 1. This strain, together with others, differs from traditional pv. savastanoi isolates in the amount of IAA produced, pathogenicity and physiological characters. Thus, its characterization was not included in this work and will be the object of further studies. Bacteria were purified on solid medium containing $\left(\mathrm{g}^{-1}\right)$ : beef extract, 3; peptone, 5; sucrose, 50; agar, 15 ; and were stored at $4{ }^{\circ} \mathrm{C}$ on a similar medium in which glycerol $(20 \mathrm{~g})$ was substituted for sucrose.

Plasmid isolation, restriction endonuclease digestion and electrophoresis. The protocols of plasmid isolation, restriction endonuclease digestion, and electrophoresis have been described (Comai \& Kosuge, 1982).

Isolation of $\mathrm{Iaa}^{-}$mutants. To isolate spontaneous $\mathrm{Iaa}^{-}$mutants bacteria were grown in glucose/peptone broth (Comai \& Kosuge, 1982) to late exponential phase, washed in $10 \mathrm{~mm}$-saline, and serially diluted on minimal medium A (Miller, 1972) to which $\alpha$-methyltryptophan $\left(150 \mu \mathrm{g} \mathrm{ml}^{-1}\right)$ had been added after autoclaving. The plates were incubated at $23{ }^{\circ} \mathrm{C}$ for $5 \mathrm{~d}$ at which time two colonies per strain were picked, purified twice and stored on agar slants at $4{ }^{\circ} \mathrm{C}$ for later characterization.

Determination of IAA production. This followed the procedure of Comai \& Kosuge (1980).

In vitro labelling of DNA and Southern blot hybridization. DNA was labelled in vitro by 'nick translation' with Escherichia coli DNA polymerase I according to Rigby et al. (1977). Southern blot hybridization (Southern, 1975) followed the protocol of Davis et al. (1980) and used nitrocellulose filters (BA85) from Schleicher and Schuell, Keene, New Hampshire. Hybridization was carried out in $50 \%$ (v/v) formamide at $42{ }^{\circ} \mathrm{C}$.

Biochemicals. Agarose HGT was purchased from Marine Colloids, Rockland, Maine; restriction endonucleases from Miles Biochemicals, Eckhart, Indiana, and New England Biolabs, Beverly, Massachusetts; dNTPs and DNAase I from Boehringer Mannheim; Escherichia coli DNA polymerase I from Bethesda Research Lab, Bethesda, Maryland; all other chemicals from Sigma and Calbiochem-Behring.

\section{RESULTS}

Plasmids in pv. savastanoi strains. Plasmid DNA was isolated from each strain of pv. savastanoi and characterized by agarose gel electrophoresis. Figure. 1(a) shows the electrophoretic pattern of plasmid DNA. Four to seven plasmid species were resolved in each strain ranging in size from 7 to $100 \mathrm{~kb}$. All Italian isolates were conspicuous for the number of plasmid species when compared with Californian strains EW 1006 and EW2009 which are representative of many Californian isolates.

Relation between $\alpha$-methyltryptophan resistance and IAA production. To isolate Iaa ${ }^{-}$ mutants we used selection for $\alpha$-methyltryptophan resistance as described by Smidt \& 


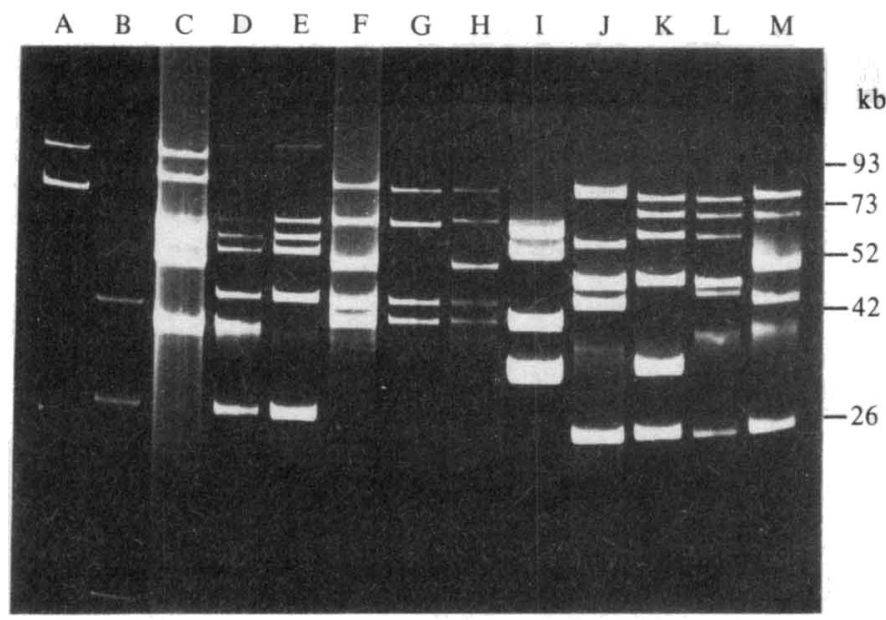

$\begin{array}{llllllllllllll}\text { E } & \text { F } & \text { G } & \text { H } & \text { I } & \text { J }\end{array}$

(a)

Fig. 1. (a) Agarose gel electrophoresis of plasmid DNA purified from pv. savastanoi strains. Olive strains: A, EW1006; B, PB207; C, PB202; D, PB230; E, PB231. Oleander strains: F, PB205; G. PB204; H, PB213; I, EW2009. Privet strains: J, PB209; K, PB215, L, PB217; M, PB218. On the right are marked the estimated sizes $(\mathrm{kb})$ of the plasmids from PB207. These were calculated from their electrophoretic mobilities in relation to the following standards: RSF1010 (8.3 kb), RSF1010::Tn1 (13.3 kb), pLUC1::Tn1 (16 kb) and RK2 (57 kb).

(b) Plasmid DNA from the gel in Fig. 1 (a) was transferred to a nitrocellulose filter after breakage by depurination in $0.25 \mathrm{M}-\mathrm{HCl}$ and hybridized to ${ }^{32} \mathrm{P}$-labelled plasmid pLUC1 using the conditions specified in Methods. Lane designations as in $(a)$. Lanes A to D are not shown since no components hybridized with the probe.

Kosuge (1978). To test the feasibility of this technique for the isolation of $\mathrm{Iaa}^{-}$mutants from different strains, we tested fifty $\alpha$-methyltryptophan resistant mutants obtained from each of strains EW2009, EW2015 and TK1050. All those derived from strains EW2009 and TK1050 were Iaa- mutants; derivatives of EW2009 all had lost plasmid pIAA1, while no changes were seen in the plasmids of $\mathrm{Iaa}^{-}$strains derived from TK1050. In the latter strain, genes for IAA synthesis are chromosomal (L. Comai, unpublished results). In contrast to derivatives of strains EW2009 and TK 1050, most of the $\alpha$-methyltryptophan resistant mutants of strain EW2015 were still IAA producers. EW2009 and EW2015 are closely related oleander strains since they harbour identical plasmids. However, EW2015 is non-fluorescent on glucose/peptone medium. The production of a water-soluble fluorescent pigment is typical of members of the $P$. syringae group (Leisinger \& Margraff, 1979).

Further, we tested ten Iaa ${ }^{-}$mutants, which had been isolated other than by $\alpha$ methyltryptophan resistance (Comai \& Kosuge, 1980) and all these were $\alpha$-methyltryptophan resistant. In addition, when plasmid pIAA 1 was reintroduced by transformation, sensitivity to $\alpha$-methyltryptophan was regained. We concluded that in strain EW2009, and other fluorescing strains, loss of IAA production always resulted in increased resistance to $\alpha$-methyltryptophan.

Isolation of Iaa $\mathrm{I}^{-}$mutants. $\alpha$-Methyltryptophan resistant mutants were isolated from each of the Italian strains, with the exception of PB204 which was already resistant to this analogue. Mutants appeared at frequencies ranging from $10^{-5}$ to $10^{-6}$. When tested for IAA production all the mutants and PB204 were negative. When inoculated in olive plants, Iaamutants did not induce galls, while the wild-types, with the exception of PB204, produced the knot disease (G. Surico \& L. Comai, unpublished observations). 


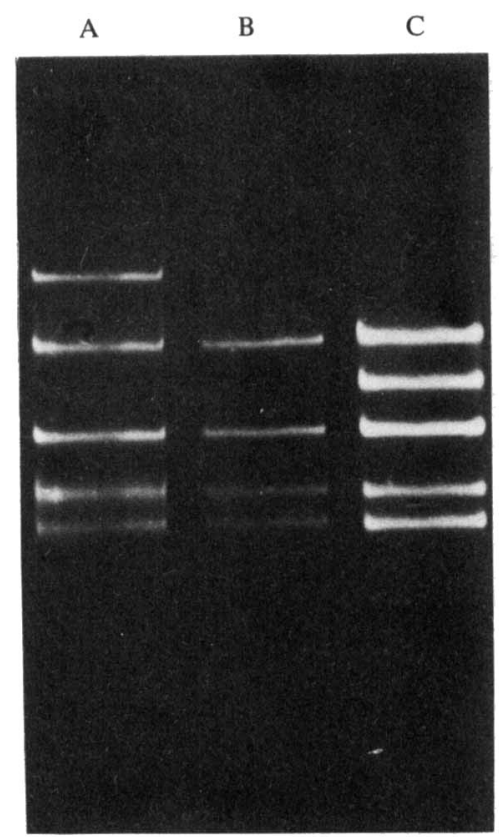

Fig. 2. Agarose gel electrophoretic analysis of plasmid DNA from wild-type strain PB213 (A) and its laa- derivatives PB213-1 (B) and PB213-2 (C).

Analysis of plasmid DNA in Iaa- mutants. Plasmid DNA was isolated from each of two mutants and compared with that of the wild-type by agarose gel electrophoresis. Of the eleven strains studied, two, PB205 and PB213, lost a plasmid, or part of it, concurrently with IAA production. Figure 2 shows the plasmid pattern of wild-type strain PB213, and of two Iaaderivatives. Both lacked the largest plasmid, $73 \mathrm{~kb}$. One laa- mutant, however, showed a new plasmid species, $50 \mathrm{~kb}$. Identical events were observed in $\mathrm{Iaa}^{-}$mutants derived from PB205. Since we postulated that the $73 \mathrm{~kb}$ plasmid coded for IAA production, we called it pIAA2.

No changes from the respective wild types were detected in the plasmids of the Iaamutants isolated from the remaining strains.

Identification of the plasmid coding IAA production by hybridization. To confirm the results from plasmid analysis we determined which plasmids in the eleven Italian and two Californian strains contained sequences homologous to the iaaM locus. In vitro ${ }^{32} \mathrm{P}$-labelled plasmid pLUC1, which contains fragment EcoRI-M of pIAA1 coding iaaM, was hybridized to the plasmids shown in Fig. 1 (a) by Southern blot hybridization. Strains PB205, PB213, and, as expected, EW2009 exhibited pLUC1 homologous sequences (Fig. 1b). The difference in bound radioactivity between pIAA 1 and pIAA2 is due to the different amount of plasmid DNA. Two additional bands visible in the tracks of PB205 and EW2009 do not correspond to any of the other plasmids and may represent linear forms of pIAAl and pIAA2. By longer exposures a similar band was also detected in the track of PB213.

Restriction endonuclease analysis of plasmids in oleander isolates. The results with strains PB205 and PB213 indicate that plasmid pIAA2 bears the genes coding for IAA production. A plasmid of similar size was present in the IAA-non-producing strain PB204, but did not hybridize to the probe plasmid pLUC 1 . To determine if pIAA2 had any relationship with pIAA1 and with the cryptic plasmid in strain PB204, plasmid DNA from strain PB213, from its two Iaa ${ }^{-}$derivatives, PB213-1 and PB213-2 (Fig. 2), and from strains PB204 and 


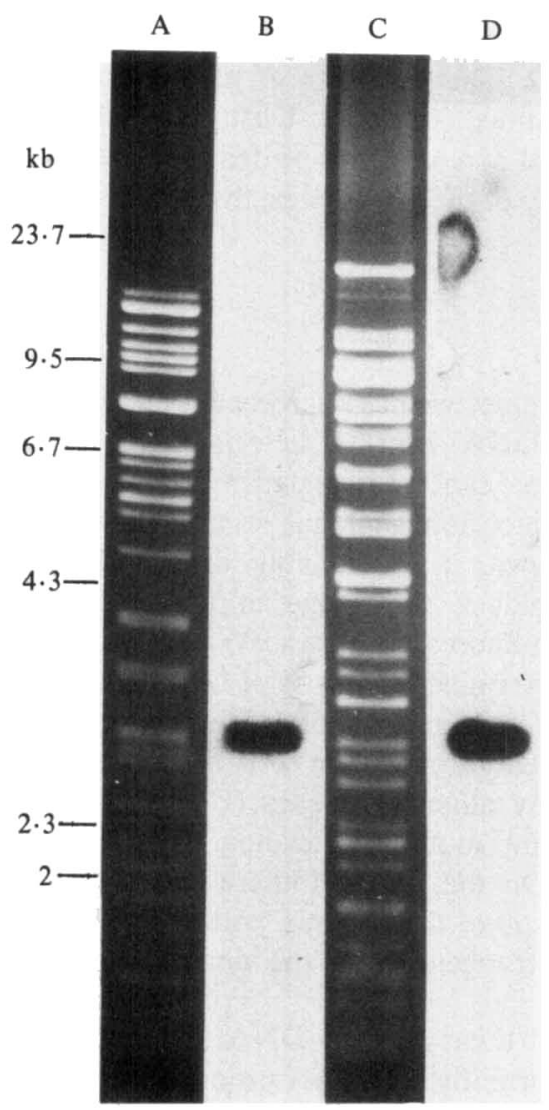

Fig. 3

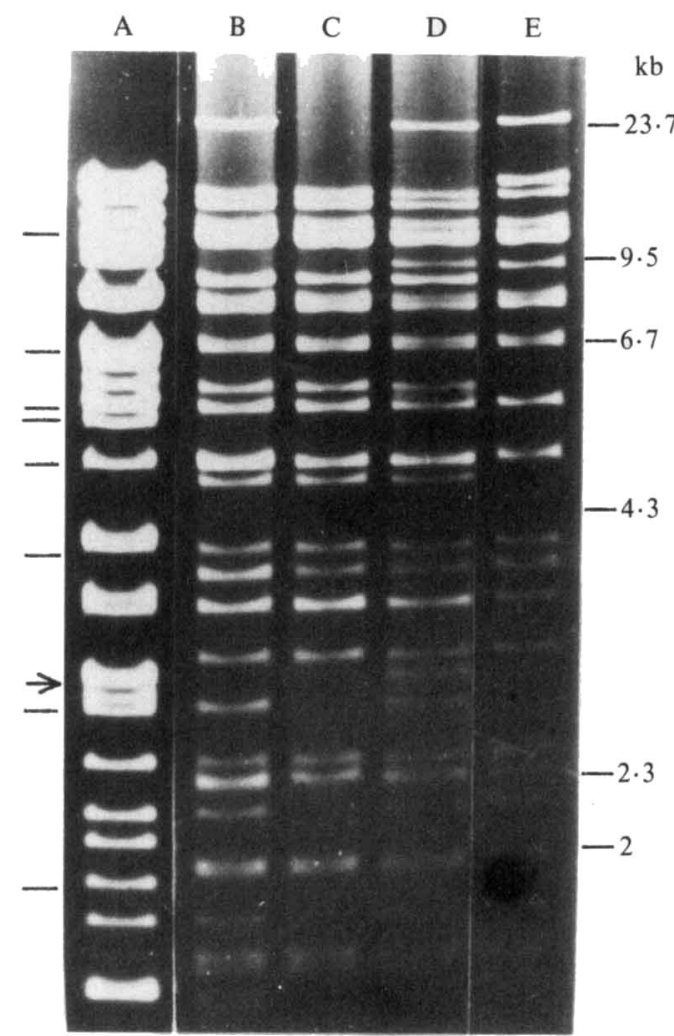

Fig. 4

Fig. 3. Restriction endonuclease EcoRI-generated fragments of plasmid DNA from strain EW2009 (A) and PB213 (C) were separated by agarose gel electrophoresis and transferred to a nitrocellulose filter (B and $\mathrm{D}$, respectively) and hybridized to ${ }^{32} \mathrm{P}$-labelled plasmid pLUC1 using the conditions specified in Methods.

Fig. 4. Agarose gel electrophoresis of EcoRI restriction endonuclease-treated plasmid DNA from the wild-type, $\mathrm{Iaa}^{+}$, strain EW2009 (A), Iaa- mutants PB213-2 (B) and PB213-1 (C), wild-type, Iaa ${ }^{+}$, PB213 (D), and the Iaa- strain PB204 (E). The lines on the left of track (A) mark the fragments of DNA belonging to pIAA1 (Comai \& Kosuge, 1980); the arrow indicates the position of the fragment which binds to pLUC1.

EW2009 (Fig. 1), was treated with restriction endonuclease EcoRI. The resulting fragments were separated by agarose gel electrophoresis and transferred to a nitrocellulose filter for hybridization to pLUC1. It was found that pLUC1 hybridized to the homologous fragment of pIAA1 and to a fragment of PB213 plasmid DNA having the same molecular weight (Fig. 3). That segment was part of pIAA2 since it was missing in the digests of PB213-1 and PB213-2, the two derivatives lacking pIAA2 (Fig. 4). The restriction fragments generated by digestion of pIAA2 can be identified by comparing the patterns of the wild-type, PB213 with those of the pIAA2-less derivative PB213-1. PB213-2 contains some of the fragments of pIAA2 and thus harbours a deletion derivative of pIAA2, here called pIAA2 $\Delta 1$. The fragments of pIAA2 constituting pIAA2 $\Delta 1$ can be identified by comparing the three patterns. PB204 harboured a plasmid closely related to pIAA2, as demonstrated by the presence in PB204 of most EcoRI fragments belonging to pIAA2, but lacking homology to EcoRI-M since no 
hybridization with pLUC1 was evident (data not shown); it was called pIAA2 22 on the assumption that it is a deletion derivative of pIAA2. The segments of pIAA1 formed by EcoRI restriction digestion have been previously identified (Comai \& Kosuge, 1980) and are marked on Fig. 4. From the EcoRI digestion pattern of pIAA2 it can be deduced that most of the homology with pIAA 1 is confined to the proximity of the IAA genes, the remainder being apparently unrelated to pIAA1.

\section{DISCUSSION}

By the use of combined genetic and physical techniques we have screened a number of pv. savastanoi strains for the presence of plasmid DNA bearing determinants for IAA production. Spontaneously occurring $\mathrm{Iaa}^{-}$mutants can be isolated from a wild-type population by their increased resistance to the tryptophan analogue $\alpha$-methyltryptophan. Although the mechanism of resistance is not known, it is probably due to the larger tryptophan pools exhibited by $\mathrm{Iaa}^{-}$mutants (M. Smidt \& $\mathrm{T}$. Kosuge, unpublished results). The reason this relationship is not observed in the non-fluorescent strain EW2015 is unknown. Using strain EW2009, which harbours pIAA1, we determined that loss of IAA production is always associated with loss of the plasmid or insertional inactivation of the IAA genes (L. Comai \& T. Kosuge, unpublished results); also, $\alpha$-methyltryptophan resistance is associated with all $\mathrm{Iaa}^{-}$mutants, since $\mathrm{Iaa}^{-}$mutants isolated by other techniques (Comai \& Kosuge, 1980) are all resistant to $\alpha$-methyltryptophan. Point mutations leading to loss of IAA production were never found in strain EW2009. On the basis of these observations we speculate that in any fluorescent strain of pv. savastanoi the plasmid coding for IAA production could be identified by its absence or rearrangement in the corresponding Iaa $^{-}$ mutants.

$\mathrm{Iaa}^{-}$mutants were isolated from each strain and their plasmid DNA analysed. In two strains, PB205 and PB213, a plasmid was lost concurrently with IAA production. When the plasmids from all strains were tested for sequences homologous to the IAA genes, only the $73 \mathrm{~kb}$ plasmid (pIAA2) present in PB205 and PB213, showed homology. The other oleander strain PB204, which had lost the capacity for IAA production during repeated subculture, harboured a plasmid closely related to pIAA2, but which had a deletion in the IAA region. Thus, all virulent oleander strains so far tested bear their IAA genes on a plasmid. All olive and privet strains tested do not, although the possibility that some of these strains harbour a very large plasmid which was not isolated by the technique used, cannot be excluded.

The relationship between pIAA1 and pIAA2 is not clear. Although the regions associated with IAA production are very similar in both plasmids as shown by EcoRI endonuclease hydrolysis and blot hybridization, the remainder of the plasmids show different EcoRI restriction patterns. This problem is currently being investigated together with the organization of the IAA genes on the chromosome (or large plasmids) of the other strains.

This work is an additional step toward the understanding of the organization of genes for virulence in phytopathogenic pseudomonads. This group of parasitic bacteria conceivably evolved from saprophytic pseudomonads, substituting catabolic versatility for capacity to parasitize plants (Misaghi \& Grogan, 1969). Production of toxins, indoleacetic acid and probably cytokinins, contribute to virulence and ultimately to parasitic fitness. The evolution of virulence is a matter of conjecture; however, there is a remarkable similarity between the pathway of catabolism of L-lysine by Pseudomonas fluorescens (Takeda \& Hayaishi, 1966) and synthesis of IAA from L-tryptophan by pv. savastanoi (Kosuge et al., 1966). Whatever the origin of the genes for IAA production among strains of pv. savastanoi, there is a considerable diversity as to where these genes are located. This heterogeneity may reflect the nature of these genes. Although Iaa ${ }^{-}$mutants can grow in the host (Smidt \& Kosuge, 1978), they cannot stimulate formation of galls, which otherwise could provide an ideal environment for the multiplication of the parasite. Thus, the IAA genes could confer a marked selective 
advantage and in this capacity resemble genes for antibiotic resistance. The capacity of these genes to become associated with different replicons would help transmission of an evolutionary advantageous trait among the members of a population. The molecular events leading to the presence of the IAA genes on plasmids and chromosome could be similar to $\mathrm{F}^{\prime}$ and $\mathrm{Hfr}$ formation in $E$. coli or to transposition of genetic elements. Studies are now in progress to characterize the system.

Preliminary pathogenicity studies have shown that in olive and privet strains IAA production is necessary for gall development (G. Surico, unpublished results) as has been reported for oleander strains (Smidt \& Kosuge, 1978). So far, in isolates from California and southern Italy only strains isolated from oleander harbour a plasmid coding for IAA production. In olive and privet isolates thus far examined, the genes for IAA production are chromosomal.

In summary, a variety and number of plasmid species were observed in strains of pv. savastanoi, from southern Italy. In the oleander strains a plasmid coding for IAA production was identified by analysing $\mathrm{Iaa}^{-}$mutants and by the presence of sequences homologous to the iaaM locus. Plasmids in the remaining strains from olive and privet exhibited neither rearrangements upon loss of IAA production, nor hybridization to the probe DNA. The new IAA plasmid, called pIAA2, did not resemble pIAA1 in its EcoRI endonuclease fragments except in the region bearing the IAA genes.

Supported in part by Grant 59-2063-1-1-643-0 from the Competitive Research Grants Office of the United States Department of Agriculture, Science and Education Administration.

\section{REFERENCES}

Beltra, R. (1959). El acido beta-indoleacetic y los tumores vegetales de origen bacteriano. Revista latinoamericana de microbiologia 2, 23-32.

Bottalico, A. \& Ercolani, G. L. (1971). Pseudomonas savastanoi (E. F. Smith) Stevens su ligustro giapponese in Puglia. Phytopathologia mediterranea $10,132-135$.

Comal, L. \& Kosuge, T. (1980) Involvement of plasmid deoxyribonucleic acid in indoleacetic acid synthesis in Pseudomonas savastanoi. Journal of Bacteriology 143, 950-957.

Comal, L. \& Kosuge, T. (1982). Cloning and characterization of $i a a M$, a virulence determinant of Pseudomonas savastanoi. Journal of Bacteriology 149, 40-46.

Davis, R. W., Botstein, D. Roth, J. R. (1980). Advanced Bacterial Genetics. New York: Cold Spring Harbor Laboratory.

HiRsch, P. R., VAN Montagu, M., Johnston, A. W. B., BrewIN, N. J. \& Schell, J. S. (1980). Physical identification of bacteriocinogenic, nodulation and other plasmids in strains of Rhizobium leguminosarum. Journal of General Microbiology $120,403-412$.

Kosuge, T., Heskett, M. G. \& Wilson, E. E. (1966). Microbial synthesis and degradation of indole-3acetic acid. I. The conversion of L-tryptophan to indole-3-acetamide by an enzyme system from Pseudomonas savastanoi. Journal of Biological Chemistry 241, 3738-3744.

Leisinger, T. \& MargrafF, R. (1979). Secondary metabolites of the fluorescent pseudomonads. Microbiological Reviews 43, 422-442.

MILleR, J. H. (1972). Experiments in Molecular Genetics. New York: Cold Spring Harbor Laboratory.
MisaghI, I \& Grogan, R. C. (1969). Nutritional and biochemical comparisons of plant-pathogenic and saprophytic fluorescent pseudomonads. Phytopathology 59, 1436-1450.

Rigby, P. W. J., DieckmanN, M., Rhodes, C. \& BERG, P. (1977). Labeling deoxyribonucleic acid to high specific activity in vitro by nick translation with DNA polymerase I. Journal of Molecular Biology 113, 237-251.

Smidt, M. \& Kosuge, T. (1978). The role of indole 3-acetic acid accumulation by alpha-methyltryptophan-resistant mutants of Pseudomonas savastanoi in gall formation on oleanders. Physiological Plant Pathology 13, 203-214.

SOUTHERN, E. M. (1975). Detection of specific sequences among DNA fragments separated by gel electrophoresis. Journal of Molecular Biology 98, 503-517.

Surico, G., Sparapano, L., Lerario, P., Durbin, R. D. \& IACOBELlIS, N. (1975). Cytokinin-like activity in extracts from culture filtrates of Pseudomonas savastanoi. Experientia 31, 929-930.

TAKEDA, H. \& HayAishi, O. (1966). Crystalline L-lysine oxygenase. Journal of Biological Chemistry 241, 2733-2736.

Watson, B., Currier, T. C., Gordon, M. P., Chilton, M. \& Nester, E. W. (1975). Plasmid required for virulence of Agrobacterium tumefaciens. Journal of Bacteriology 123, 255-264.

Wilson, E. E., HesketT, M. G., Johnson, M. L. \& Kosuge, T. (1972). Metabolic behavior of Pseudomonas savastanoi isolates from olive and oleander on certain carbohydrate and amino substrates. Phytopathology 62, 349-355. 